\title{
Archivos \\ de Cardiología de México

\section{Monitor de señales de electrocardiografía y frecuencia cardiaca mediante un teléfono móvil con el protocolo de comunicación Bluetooth}

\author{
Brayans Becerra-Luna ${ }^{a}$, Rodrigo Dávila-García ${ }^{\mathrm{b}}$, Paola Salgado-Rodríguez ${ }^{\mathrm{b}}$, \\ Raúl Martínez-Memije ${ }^{a}$ y Óscar Infante-Vázquez ${ }^{\text {a,* }}$
}

a Departamento de Instrumentación Electromecánica, Instituto Nacional de Cardiología Ignacio Chávez, México D.F., México

b Departamento de Ingeniería Biomédica, Instituto Tecnológico y de Estudios Superiores de Monterrey Campus Ciudad de México, México D.F., México

Recibido el 21 de diciembre de 2011; aceptado el 17 de abril de 2012

\author{
PALABRAS CLAVE \\ Java 2 Micro Edition; \\ MOBILE 0007; \\ PIC18F2550; \\ Electrocardiografía \\ en celular; \\ Bluetooth; \\ México
}

\begin{abstract}
Resumen
Objetivo: Desarrollar un equipo de monitoreo de señales de electrocardiografía (ECG) y frecuencia cardiaca ( $\mathrm{FC}$ ) portátil, comunicado con un teléfono móvil mediante el protocolo de comunicación Bluetooth (BT), para su visualización en pantalla.

Métodos: Se diseñó un sistema de monitoreo que en su sección electrónica realiza la adquisición de la señal de ECG, así como su amplificación, filtrado, conversión analógica a digital y transmisión por BT del ECG y la FC. Se desarrollaron dos programas para el sistema. El primero calcula la FC a través de la identificación del QRS, y envía las señales del ECG y FC al teléfono móvil. El segundo es una aplicación que permite adquirirlas y visualizarlas en la pantalla del móvil.

Resultados: Se desarrolló un sistema electrónico portátil alimentado por una batería de 9 volts, de amplificación y ancho de banda que cumplen con los estándares internacionales, para monitoreo de ECG. La identificación del complejo QRS se realizó con el algoritmo de la segunda derivada, mientras que los programas realizados permiten enviar y recibir la información del ECG y la FC a través de BT, para visualizarlos en la pantalla del móvil. El monitoreo es factible hasta $15 \mathrm{~m}$ de distancia. Se probó en distintos móviles de Nokia ${ }^{\circledR}$, Sony Ericsson ${ }^{\circledR}$ y Samsung ${ }^{\circledR}$. Conclusiones: Este sistema muestra una alternativa de monitoreo móvil, con un teléfono por medio de BT y la programación en Java 2 Micro Edition (J2ME). Permite registrar el trazo del ECG y la FC, y puede implementarse en diferentes móviles.

(C) 2011 Instituto Nacional de Cardiología Ignacio Chávez. Publicado por Masson Doyma México S.A. Todos los derechos reservados.
\end{abstract}

\footnotetext{
* Autor para correspondencia: Departamento de Instrumentación Electromecánica. Instituto Nacional de Cardiología Ignacio Chávez. Juan Badiano 1, Colonia Sección XVI, Delegación Tlalpan. C.P. 14080, México D.F., México. Tel.: +52 (55) 5573 2911, extensión 1386; Fax: +52 (55) 55730926.

Correo electrónico: infosc@cardiologia.org.mx (Ó. Infante-Vázquez).
} 


\section{KEYWORDS}

Java 2 Micro Edition;

MOBILE 0007;

PIC18F2550;

Electrocardiography

in cellular;

Bluetooth;

Mexico

\section{Monitor of ECG signal and heart rate using a mobile phone with Bluetooth} communication protocol

\begin{abstract}
Objective: To develop a portable signal monitoring equipment for electrocardiography (ECG) and heart rate (HR), communicated with a mobile phone using the Bluetooth (BT) communication protocol for display of the signal on screen.

Methods: A monitoring system was designed in which the electronic section performs the ECG signal acquisition, as well as amplification, filtering, analog to digital conversion and transmission of the ECG and HR using BT. Two programs were developed for the system. The first one calculates HR through QRS identification and sends the ECG signals and HR to the mobile, and the second program is an application to acquire and display them on the mobile screen.

Results: We developed a portable electronic system powered by a 9 volt battery, with amplification and bandwidth meeting the international standards for ECG monitoring. The QRS complex identification was performed using the second derivative algorithm, while the programs allow sending and receiving information from the ECG and HR via BT, and viewing it on the mobile screen. The monitoring is feasible within distances of $15 \mathrm{~m}$ and it has been tested in various mobiles telephones of brands Nokia ${ }^{\circledR}$, Sony Ericsson ${ }^{\circledR}$ and Samsung ${ }^{\circledR}$.

Conclusions: This system shows an alternative for mobile monitoring using BT and Java 2 Micro Edition (J2ME) programming. It allows the register of the ECG trace and HR, and it can be implemented in different phones.

(c) 2011 Instituto Nacional de Cardiología Ignacio Chávez. Published by Masson Doyma México S.A. All rights reserved.
\end{abstract}

\section{Introducción}

El aumento en la longevidad y en las enfermedades cardiovasculares en nuestra población, impulsan a acentuar el cuidado de la salud ${ }^{1}$. Esto lleva a un aumento en los costos de la atención a la salud de este grupo poblacional, por lo que sería conveniente contar con soluciones tecnológicas con las cuales se pueda dar un seguimiento a este tipo de patologías, por este motivo se hace necesario un automonitoreo fácil y sobre todo confiable. El monitoreo de signos vitales mediante sistemas portátiles y su integración a equipos de telefonía móvil o computadoras personales, da como resultado una mejor calidad de vida y autocuidado, lo que depende en gran medida de la habilidad para compartir la información clínica del paciente en tiempo real ${ }^{2}$. Por esto, distintos grupos de investigación, como los de la Universidad Tecnológica de Malasia, han desarrollado una interfaz que ha sido probada para la transferencia de los datos de los dispositivos médicos de signos vitales a un sistema de procesamiento de datos, a través de una red inalámbrica ${ }^{3}$.

Como se sabe, algunas variables fisiológicas básicas que permiten conocer el estado de salud de un sujeto son la frecuencia cardiaca (FC) y la señal del electrocardiograma (ECG).

La FC se define como el número de veces que el corazón se contrae en un minuto, expresado en latidos por minuto $(\mathrm{lpm})^{4}$, en tanto, el ECG es la representación gráfica de la actividad eléctrica del corazón, lo que constituye un instrumento principal de la electrofisiología cardíaca y tiene una función relevante en la identificación y diagnóstico de las enfermedades cardiovasculares ${ }^{5}$.

El monitoreo de la señal del ECG y de la FC se puede realizar de diferentes maneras, una de ellas es a través de equipos de electrocardiografía como: electrocardiógrafos o grabadoras Holter ${ }^{6}$. En tanto que los electrocardiógrafos son equipos estáticos utilizados en la clínica, los Holters son grabadoras de la señal del ECG de largo tiempo, con los que se obtiene un registro de ésta, incluso al realizar actividades físicas.

Existen también equipos para monitoreo de la FC mediante telefonía móvil, entre ellos uno creado en conjunto por Nokia $^{\circledR}$ y Polar $^{\circledR}$, dirigido al modelo Nokia N79 ${ }^{\circledR 7}$, este monitor se comunica a través de Bluetooth (BT) y requiere de un equipo de amplificación de la señal de ECG. La aplicación desarrollada para el monitoreo es de uso exclusivo para este móvil, por ser de gama alta. También Apple Computers $^{\circledR}$ desarrolló un sistema para monitoreo de FC en teléfono móvil, haciendo uso de su iPhone ${ }^{\circledR 8}$ y de los elementos internos con los que cuenta este dispositivo, como su acelerómetro. En ambos casos estas aplicaciones sólo funcionan para dichos móviles.

En modelos de investigación también se tienen sistemas capaces de conectarse a través del protocolo de comunicación BT y enviar así información sobre la FC al teléfono móvil, teniendo conexión a través del Servicio General de Paquetes vía Radio (GPRS, por sus siglas en Inglés) para contactar con el médico ${ }^{9}$, o poder mostrar un ECG de forma continua en el móvil ${ }^{10}$.

Considerando lo anterior, nuestro grupo se abocó al desarrollo de un equipo capaz de realizar el monitoreo de la frecuencia cardiaca y el despliegue de la señal electrocardiográfica en un teléfono móvil mediante la comunicación inalámbrica, utilizando para ello el protocolo de comunicación BT, que se encuentra disponible en diferentes marcas de móviles no necesariamente de gama alta.

El equipo que aquí se propone utiliza el protocolo de comunicación BT, mismo que se ha empleado como una plataforma atractiva para diversas aplicaciones médicas, y que 
viene integrado en teléfonos celulares de gama media, el que siendo un protocolo de comunicación en redes de área personal, está diseñado para comunicación en distancias cortas $^{11}$. Dentro de las principales ventajas de nuestro sistema está la flexibilidad de utilizarse con un número mayor de teléfonos celulares, esto es debido a que hace uso de la máquina virtual de Java, que viene precargado en este tipo de móviles. De manera que no se vuelve exclusivo de una sola marca de móviles o modelos de dichos teléfonos.

El equipo aquí propuesto permite monitorear el ECG y la FC con las especificaciones de las normas internacionales, en lo correspondiente a los anchos de banda de la señal del ECG y la seguridad eléctrica del paciente ${ }^{12}$. En cuanto a la detección del complejo QRS y el cálculo de la FC, este se realiza mediante el método de la segunda derivada, el cual ya ha sido validado con anterioridad ${ }^{13}$, y asimismo cumple con las especificaciones del caso $^{14}$.

Dicho equipo se ha diseñado para operar en condiciones de movimiento, de manera que el usuario del sistema pueda realizar actividades físicas.

En cuanto al medio de comunicación, las especificaciones de BT determinan la operación del equipo que utiliza un módem BT clase II, las cuales establecen una distancia de operación máxima de $10 \mathrm{~m}$. Una limitación importante de este tipo de comunicación es el consumo de corriente, que es superior a los $100 \mathrm{~mA}$ mientras transmite ${ }^{11}$.

\section{Métodos}

Para el desarrollo del sistema de monitoreo de señales del ECG y FC, se procedió como lo indica el diagrama a bloques de la figura 1, el cual muestra las partes más significativas para el diseño del monitor de señales del ECG y FC que son:

a) Módulo de adquisición de la señal del ECG.

b) Digitalización y procesamiento del ECG.

c) Transmisión de datos del ECG y de la FC.

d) Recepción y despliegue del ECG y de la FC.

\section{Adquisición de señal del ECG}

Para la obtención analógica de la señal del ECG se requieren básicamente de dos etapas electrónicas: amplificación y filtrado de la señal.

Todo el sistema es alimentado por una batería de 9 volts, la cual va conectada a un circuito convertidor de corriente directa (DC, por sus siglas en Inglés), con salidas de voltaje positivo y negativo, que dará una salida de -9 volts, ya que los amplificadores operacionales de uso general empleados en este sistema son del tipo JFET de bajo consumo de energía, de la familia TL074 de Texas Instruments ${ }^{\circledR}$, los cuales operan con voltajes positivos y negativos, de acuerdo con la configuración que se utilizó.

El sistema tiene un cable de paciente para la toma del ECG, cuyas terminales se colocan en el sujeto en la configuración para el registro de la derivación $\mathrm{DI}$ y al sistema, a través de las entradas de un amplificador de instrumentación AD620 de la empresa Analog Devices ${ }^{\circledR}$, ajustado a una ganancia $(G)=20$.

La salida de dicho amplificador se conecta a un TL074 en configuración para efectuar un filtrado pasa altas de segundo orden ${ }^{15}$, con frecuencia de corte de $0.5 \mathrm{~Hz}$, lo que reduce la posibilidad de saturación del sistema y la deriva de la línea base causada por la respiración.

La segunda sección de amplificación del sistema fue diseñada con una $G=50$, para obtener una $G$ Total $G_{T}=1$ 000 , posteriormente se conecta a un filtro pasa bajas ${ }^{15}$, con frecuencia de corte en $42.5 \mathrm{~Hz}$, que es utilizado para evitar el efecto de Nyquist por submuestreo al momento de la conversión analógica-digital de la señal ${ }^{16}$.

El circuito de pierna derecha está creado para lograr un balanceo de impedancias, con el propósito de reducir los voltajes en modo común ${ }^{17}$.

Con el propósito de ocupar todo el rango dinámico del convertidor analógico a digital del microcontrolador, que es de 0 a 5 volts, se diseñó un circuito para la elevación del nivel de voltaje en el cero de la señal, que en su origen contiene valores negativos, con un arreglo de resistencias con las cuales se puede dar un nivel de DC sumado a la señal que sale del filtro pasa bajas, que se conecta a la entrada del canal analógico 0 del microcontrolador PIC18F2550.

\section{Digitalización y procesamiento del ECG}

Para la digitalización y procesamiento del ECG se utilizó el microcontrolador PIC18F2550 ${ }^{18}$, el cual cuenta con un convertidor de señal analógica a su contraparte digital (ADC, por sus siglas en inglés) de 10 bits de resolución en amplitud, y con comunicación de tipo serial que además presenta bajo consumo de corriente. Dichas especificaciones lo vuelven óptimo para su uso en este diseño, ya que el sistema es alimentado por una batería y la comunicación serial es utilizada para el módem BT. El software utilizado para la programación del PIC, es el PIC C COMPILER Versión $4.032^{19}$.

Una vez obtenida la señal del ECG, ésta es digitalizada con el ADC interno del microcontrolador PIC18F2550 a una resolución de 8 bits y una frecuencia de muestreo de 200 muestras/segundo, lo que cumple con la regla de Nyquist ${ }^{16}$.

A continuación se enlistan los procesos efectuados por el microcontrolador para el procesamiento de la señal del ECG:

- Espera de comando de sincronización para elección de función, ECG o FC.

- Digitalización de la señal analógica a través del ADC.

- Llenado del buffer con la señal digitalizada del ECG.

- Detección del complejo QRS. Se utiliza el algoritmo del método de la segunda derivada para la localización del QRS en tiempo real ${ }^{13}$.

- Cálculo del intervalo de tiempo entre complejos QRS, para la obtención de la FC.

- Envío de paquetes de datos hacia el Módem BT, para mostrar el ECG en pantalla.

- Envío de los valores de la FC en formato ASCII hacia el Módem BT.

\section{Transmisión de datos del ECG y de la FC}

Para la transmisión de los datos del ECG y de la FC hacia el teléfono móvil se utilizó un módem BT, que se describe a continuación. 


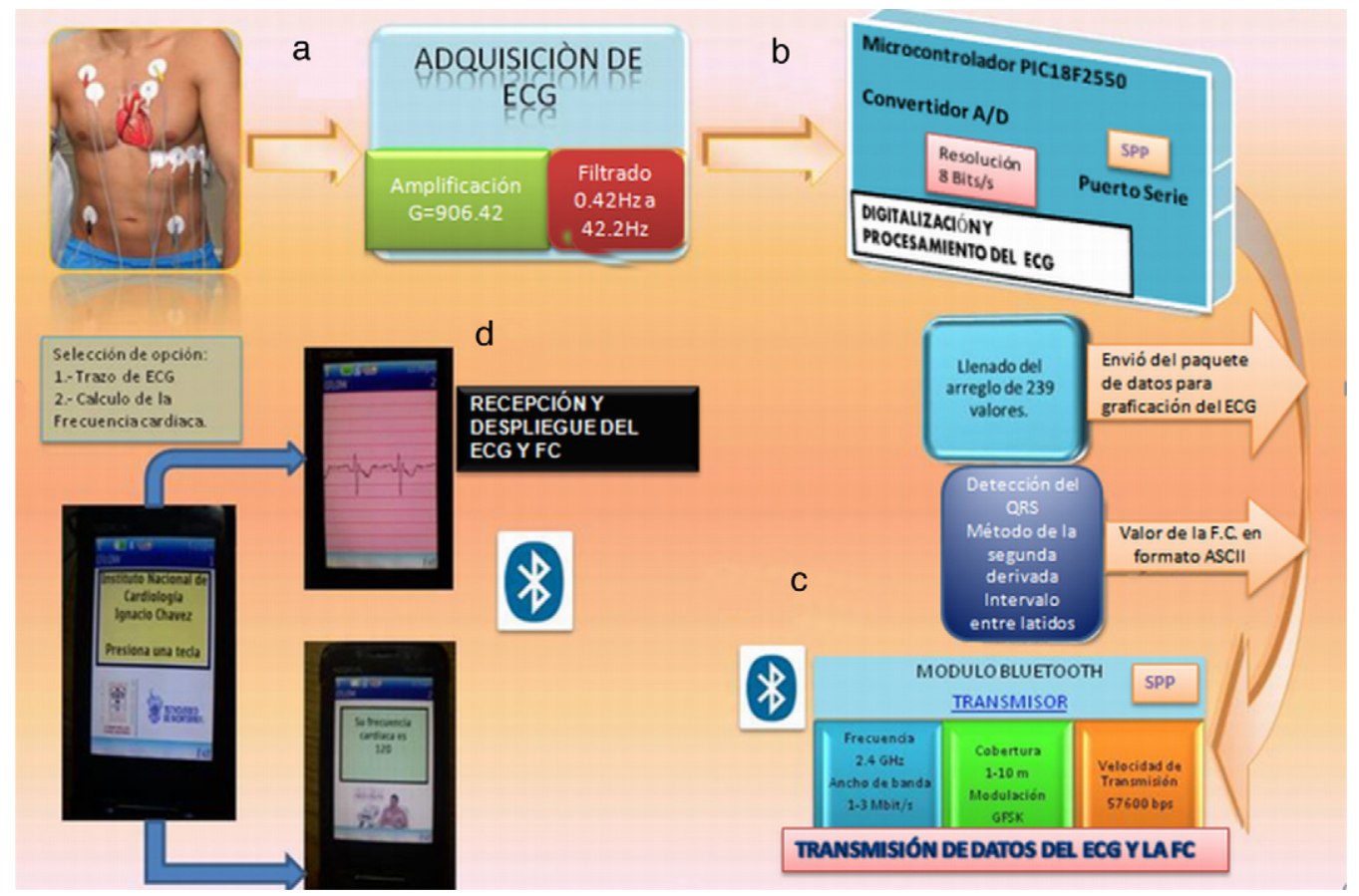

Figura 1 Diagrama a bloques del sistema de monitoreo. En este diagrama se muestran las partes que componen al sistema de monitoreo.

El módem BT utilizado es el Parani ESD-10020, éste es compatible con el Protocolo de Puerto Serie (SPP, por sus siglas en inglés), cuenta con cuatro modos distintos de programación. Para este desarrollo se utiliza el modo tres de operación, el cual es detectable para toda fuente de BT.

El microcontrolador realiza la tarea de hacer paquetes de 240 datos, los cuales contienen los valores de la señal del ECG, además de los de la FC. Dichos valores son enviados del microcontrolador al módem, y estos a su vez son enviados por el módem BT sobre una banda de frecuencia (portadora) de $2.4 \mathrm{GHz}$, que corresponde al protocolo de comunicación $\mathrm{BT}$, que tiene además modulación por desplazamiento de frecuencia gaussiana (GFSK, por sus siglas en inglés) y de acuerdo con la norma BT clase II, se comunica a un máximo de $10 \mathrm{~m}$.

\section{Recepción y despliegue del ECG y de la FC}

Para la recepción y despliegue de la señal del ECG y de la FC, se utilizó un teléfono móvil marca Nokia modelo 5610 XpressMusic ${ }^{\circledR}$ programado en Java 2 Micro Edition (J2ME) ${ }^{21}$, bajo el compilador Mobile $0007^{22}$.

Existen antecedentes de comunicación entre un móvil y dispositivos $\mathrm{BT}^{23}$, mismos que se tomaron como base para el desarrollo de la aplicación presentada.

Para la recepción y despliegue de la señal del ECG y la FC, la aplicación realiza los siguientes procesos a través de una máquina de estados:

- Inicialización de la aplicación y limpieza de la memoria de bucle.

- Búsqueda de dispositivos BT con SPP.

- Sincronización y conexión.

- Envío de orden de operación al microcontrolador.
- Recepción de datos, despliegue y actualización de ECG y FC.

\section{Pruebas de funcionamiento del sistema}

Tomando en consideración las pruebas efectuadas en la aplicación de monitoreo de FC y la detección del complejo QRS, a través de la ejecución del algoritmo de la segunda derivada en el microcontrolador, y aunque tal algoritmo ya estaba previamente validado ${ }^{13}$, se verificó el monitoreo de las señales de ECG y FC con un simulador de arritmias ${ }^{24}$, por otro lado se realizaron pruebas con un voluntario que se desplazaba por un área libre de obstáculos.

\section{Resultados}

\section{Resultados de la etapa analógica}

En la tabla 1 se presentan los resultados de las valoraciones de las características eléctricas del sistema físico, donde se aprecia que el ancho de banda del módulo de adecuación analógica de la señal de ECG cumple con la norma del caso ${ }^{12}$, mientras que los consumos encontrados son relativamente altos y por ende, sólo permiten un funcionamiento continuo del sistema durante una hora. Asimismo, la ganancia total reportada ajusta la señal al uso óptimo del rango dinámico del convertidor empleado en esta aplicación.

\section{Resultados de la etapa de digitalización y transmisión}

Una vez digitalizada la señal del ECG, el microcontrolador la almacena en un arreglo de $\mathbf{2 4 0}$ datos de tipo entero que son 
Tabla 1 Características eléctricas del sistema: voltaje, corriente, ganancia y ancho de banda

\begin{tabular}{ll}
\hline Parámetros & Mediciones obtenidas \\
\hline $\begin{array}{l}\text { Voltaje de operación } \\
\begin{array}{l}\text { Corriente total de consumo al } \\
\text { transmitir }\end{array}\end{array}$ & $9 \mathrm{~V}$ \\
$\begin{array}{l}\text { Corriente total de consumo sin } \\
\text { transmitir }\end{array}$ & $69,4 \mathrm{~mA}$ \\
$\begin{array}{l}\text { Ganancia en amplificador de } \\
\quad \text { instrumentación (G) }\end{array}$ & 19,28 \\
$\begin{array}{l}\text { Ganancia en segundo } \\
\text { amplificador (G) }\end{array}$ & 47 \\
$\begin{array}{l}\text { Ganancia total del sistema (G) } \\
\text { Ancho de banda }\end{array}$ & 906,42 \\
\hline
\end{tabular}

$\mathrm{V}$ : volts (unidad de medida de potencial eléctrico); mA: miliampers (unidad de medida de corriente eléctrica); Hz: hertz (unidad de medida para frecuencia).

enviados al módem BT, el cual realiza la tarea de cambiar la información recibida de tipo serial a una trama de BT, que será recibida por el móvil.

Para el cálculo de la FC se utilizó el algoritmo del método de la segunda derivada para detección del QRS en tiempo real ${ }^{13}$. Aunque el algoritmo ya ha sido validado para probar la efectividad de la implementación de este en dicho sistema, se introdujeron señales con distintas relaciones señal a ruido $(\mathrm{SNR})$ medidas en decibeles $(\mathrm{dB})$, desde $\mathrm{SNR}=23.4 \mathrm{~dB}$ hasta $\mathrm{SNR}=18.5 \mathrm{~dB}$, condiciones en las cuales el sistema puede realizar adecuadamente las detecciones de los complejos QRS, y por ende puede hacer el cálculo de la FC sin error apreciable.

Por otro lado, cuando se realizaron pruebas con señales de ECG a distintos latidos por minuto para determinar la cantidad de latidos perdidos en relación a la FC y el porcentaje de error, se obtuvieron los resultados que se concentran en la tabla 2. En ella se aprecia que a muy bajas frecuencias cardiacas se registra un error de cerca del 1.5\%, pero a frecuencias mayores el error respectivo fue inapreciable.

En la figura 2 se muestra la pantalla del osciloscopio donde se observa el trazo de un ECG en su derivación DI,

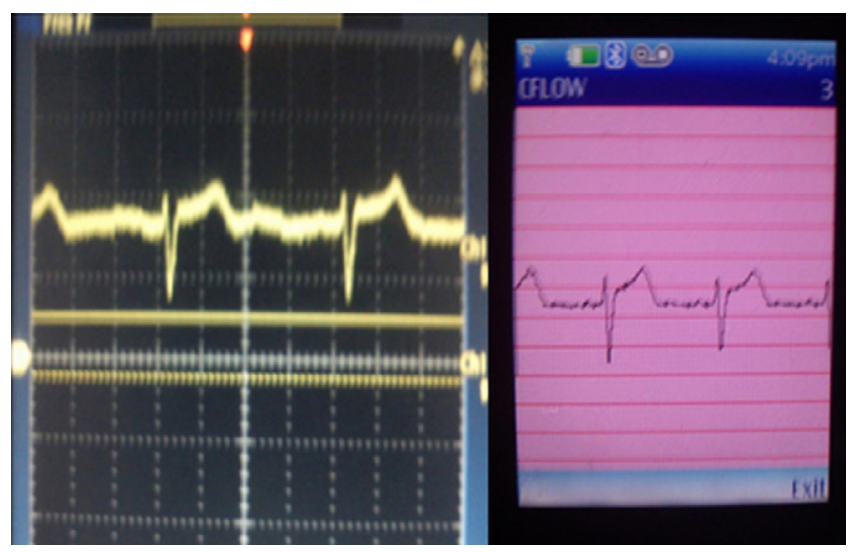

Figura 2 En el lado izquierdo de la figura se observa la señal del ECG, en un osciloscopio colocando los electrodos en DI, de lado derecho se observa la misma señal pero ahora en la pantalla del móvil. registrado simultáneamente en la pantalla del móvil. Se aprecia la alta similitud de ambos registros.

\section{Resultados obtenidos al programar el teléfono celular}

El prototipo opera a una velocidad de transmisión de 57600 baudios. En la pantalla del móvil se presenta el trazo del ECG, el cual está compuesto por 240 puntos que corresponden a la cantidad de pixeles del ancho de la pantalla, dicho trazo se actualiza cada $1200 \mathrm{~ms}$, que es el tiempo de llenado del buffer del microcontrolador. La FC mostrada en el sistema, se actualiza cada vez que se detecta un complejo QRS (fig. 3).

La duración de la batería del móvil con BT en operación es de aproximadamente tres horas. Mientras que la batería del equipo desarrollado tiene una duración continua de una hora aproximadamente.

\section{Resultados de las pruebas de funcionamiento del sistema}

Se eligieron 135 latidos para la evaluación en la detección del complejo QRS, mismos que corresponde a la cantidad máxima de datos que se pueden presentar en pantalla, con el software utilizado para dicha evaluación, de esa manera se obtuvieron los latidos reportados en la tabla 2.

Para verificar el despliegue de las señales del ECG, se realizaron pruebas de monitoreo de señales de ECG de un simulador de arritmias $^{24}$, en un área libre de obstáculos a $1 \mathrm{~m}$ de distancia, logrando mostrar en pantalla las señales de ECG introducidas con el simulador. Posteriormente, se realizó la misma prueba en idénticas condiciones pero a distancias mayores a $15 \mathrm{~m}$, de tal modo que el sistema no logra realizar una conexión por medio de $\mathrm{BT}$, debido a que excede los límites máximos de conectividad de acuerdo con la norma de BT clase II, por tanto, es nula la transmisión de datos y despliegue de la señal en la pantalla del móvil.

La aplicación desarrollada se probó en equipos Nokia ${ }^{\circledR}$ N73, N95, 6300, 5610 XpressMusic, en Sony Ericsson ${ }^{\circledR}$ P1, w810, w580 y w380, además del Samsung ${ }^{\circledR}$ S3350, siendo estos últimos incompatibles con la aplicación, debido a que no cuenta con el API JSR-82, que es el encargado del control de acceso del puerto serie virtual a través de BT.

\section{Discusión}

El desarrollo de este monitor cardiaco operable en diferentes tipos de móviles constituye un avance en el monitoreo de las señales electrocardiográficas y de la FC en forma móvil, dado que el dispositivo permitirá al usuario del sistema tener una lectura de su FC y un trazo de su señal del ECG, mientras se encuentra en actividad física o en reposo.

Cabe mencionar que el sistema desarrollado presenta ventajas y desventajas con respecto a los citados en este documento, como parte de esas ventajas tenemos:

- Un sistema versátil por la compatibilidad de conexión a través de BT. 
Tabla 2 Evaluación de la frecuencia cardiaca estimada con tres muestras de 135 latidos cada una, las cuales fueron obtenidas de un simulador de arritmias con un nivel de ruido 0 , a diferentes frecuencias cardiacas del simulador. Los valores reportados de la frecuencia cardiaca están en latidos por minuto. Los valores entre paréntesis indican el número de veces que se obtuvo esa medición de frecuencia cardiaca

\begin{tabular}{lcccrc}
\hline \multicolumn{5}{c}{ Frecuencia cardiaca obtenida } \\
\hline Muestra & FC & Máximo & Promedio & Mínimo & Latidos perdidos (\%) \\
\hline 1 & 50 & $57(30)$ & $50(89)$ & $48(14)$ & $1,48(2)$ \\
2 & 80 & $80(135)$ & $80(135)$ & $80(135)$ & $0(0)$ \\
3 & 120 & $123(4)$ & $122(92)$ & $120(39)$ & $0(0)$ \\
\hline
\end{tabular}

FC: frecuencia cardiaca del simulados.

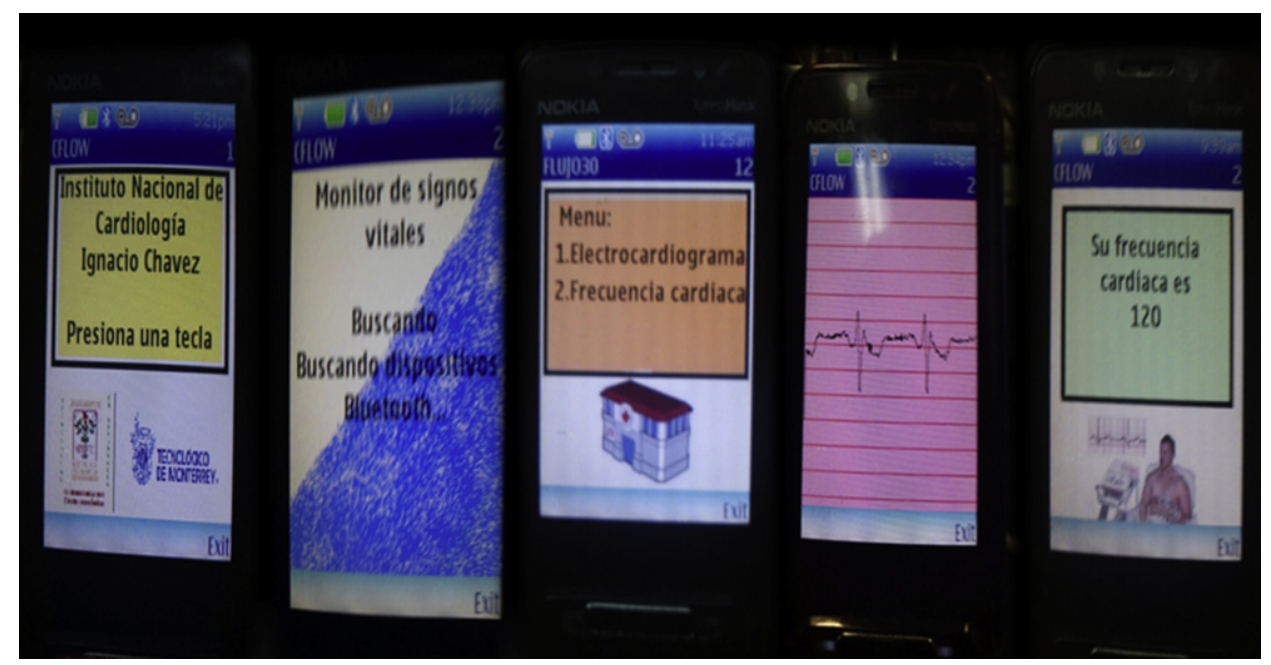

Figura 3 Pantallas que presentan la aplicación del móvil en funcionamiento. En pantalla se despliega el trazo de ECG y el valor de la frecuencia cardiaca.

- La programación en J2ME permite que esta aplicación pueda ser implantada en una mayor cantidad de dispositivos.

- Muestra trazos de ECG y la FC de manera continua.

- Las limitaciones que al momento presenta esta propuesta son las siguientes:

- No cuenta con una alarma que pueda indicar FC máxima y mínima

- No tiene programado un registro de almacenamiento de señales en el móvil.

- El consumo de energía es alto.

Dado lo anterior, este sistema nos da pie a realizar futuras mejoras como las enumeradas arriba, además de aplicaciones que no sean sólo de monitoreo, sino también de diagnóstico.

Actualmente, el desarrollo de los sistemas operativos Android y Windows Phone ha crecido aceleradamente y siguiendo la tendencia de este mercado, queda a discusión la posibilidad de desarrollar nuevas aplicaciones médicas, basadas en estos sistemas operativos o de migrar las ya existentes sobre Java Virtual Machine.

Se vislumbra la posibilidad de poder enviar el trazo de ECG y la FC a un médico o centro de monitoreo en donde, además de la evaluación realizada por el especialista, se podría elaborar una base de datos para generar conocimientos a partir de ella².

\section{Conclusiones}

El sistema que aquí se describe muestra una alternativa versátil de monitoreo móvil del ECG y de la FC, gracias a la comunicación mediante BT y la programación en J2ME, de manera que es posible registrar variables fisiológicas en la pantalla de un teléfono móvil, además se tiene la capacidad de implementarse en distintos teléfonos móviles, debido a la plataforma de programación, volviéndolo compatible con más modelos de teléfonos móviles.

Las características del sistema son las siguientes: un sistema electrónico portátil capaz de operar con una batería de 9 volts, amplificación del ECG con $\mathrm{G}$ total $\mathrm{G}_{\mathrm{T}}=906.42$ y un ancho de banda de $0.42 \mathrm{~Hz}$ hasta $42.2 \mathrm{~Hz}$. Identificación del complejo QRS, mediante el algoritmo de la segunda derivada, programado en un PIC18F2550, que hace posible comunicarse con un módem BT, que envía información de la FC y del ECG, lo que permite visualizarlos en la pantalla del móvil, dando la posibilidad de tener monitoreo a distancia con un alcance máximo de $15 \mathrm{~m}$, en espacio libre.

Adendum. Al enviar este trabajo al proceso de evaluación de la revista Archivos de Cardiología de México, nuestro grupo estaba contemplando la posibilidad de realizar la 
migración del sistema descrito a un plataforma Android, cosa en la que se avanzó durante el proceso de arbitraje, de manera que al momento de la publicación, ya se cuenta con la adecuación de la propuesta para que pueda ejecutarse en plataformas Android ver. 2.1 y se ha probado exitosamente en los siguientes móviles: Sony Ericsson ${ }^{\circledR}$ X10, X10 mini, Samsung Galaxy Ace ${ }^{\circledR}$.

Dado que las tabletas y los móviles con sistema Android comparten el uso de aplicaciones y el control de algunos elementos de hardware, se procedió a realizar pruebas de monitoreo de la FC con una Tablet Acer Iconia Tab $A 200^{\circledR}$, siendo satisfactorias dichas pruebas.

\section{Financiamiento}

No se recibió patrocinio de ningún tipo para llevar a cabo este estudio.

\section{Conflicto de intereses}

Los autores declaran no tener ningún conflicto de intereses.

\section{Referencias}

1. Lara-Esqueda A, Gómez-Montero A, Tapia-Olarte F. Programa de acción: enfermedades cardiovasculares e hipertensión arterial. 1. $\underline{a}$ ed. México: Secretaria de Salud; 2001. p. 9-10.

2. Consultado el 12 de junio de 2012. http: / / www.cenetec.salud. gob.mx/descargas/comite_e-Salud/DraVillareal22Mayo08.pdf

3. Indra HM, Eko S, Norlaili MS, et al. Wireless medical interface using zigbee and bluetooth technology. Asia. En: Third Asia International Conference on Modelling \& Simulation 2009. p. 276-81.

4. Vogel CU, Wolpert C, Wehling M. How to measure heart rate? Eur J Clin Pharmacol 2004;60:461-6.

5. Harrison RFT, Braunwald A, Kasper E. Electrocardiografía. En: Fauci AS, Braunwald E, Kasper DL, et al., editores. Principios de Medicina Interna. $17 .^{\mathrm{a}}$ ed. México: McGraw-Hill Interamericana Editores; 2006. p. 258.

6. Consultado el 12 de junio de 2012. http://www. saludalia.com/Saludalia/servlets/contenido/jsp/parserurl.jsp? url=web_saludalia/pruebas_diagnosticas/doc/doc_holter.xml

7. Consultado el 12 de junio de 2012. http://www. allaboutsymbian.com/news/item/8769_The_N79_Active_and _new_Sports_.php
8. Consultado el 12 de junio de 2012. http://www.puntofape. com/el-iphone-controlara-tu-ritmo-cardiaco-1075/

9. Thulasi Bai V, Srivatsa SK. Design and simulation of portable telemedicine system for high risk cardiac patients, India. World Academy of Science Engineering and Technology 2006: 67-71.

10. Chen X, Ho CT, Lim ET, et al. Cellular phone based online ecg processing for ambulatory and continuous detection. Comput Cardiol 2007;34:653-6.

11. Consultado el 12 de junio de 2012. http://www. seguridadmobile.com/bluetooth/especificacion-bluetooth/ estandar-bluetooth/index.html

12. Mark JB. Atlas of cardiovascular monitoring. 1. $\underline{a}$ ed. New York, USA: Churchill Livingstone; 1998. p. 130.

13. Infante $\mathrm{O}$, Valenzuela $\mathrm{F}$, Polo $\mathrm{S}$. Algoritmo que utiliza la segunda derivada para identificar el complejo QRS en tiempo real. Rev Méx Ing Bioméd 1992;13:23-32.

14. Consultado el 12 de junio de 2012. http://www. techstreet.com/cgi-bin/detail?doc_no=aami\%7Cec13_2002 _r2007_; product_id=1039641

15. Webster JG. Amplifiers and signal processing. En: Webster JG, Clark Jr JW, Neuman MR, et al., editores. Medical instrumentation application and design. $4^{\text {ta }}$ ed. USA: John Wiley and Sons Inc; 2008. p. 108-9.

16. Consultado el 13 de junio de 2012. http://cnx.org/content/ m10794/latest/

17. Consultado el 13 de junio de 2012. http://www. insumosmedicos.info/IMG/article_PDF/Electrocardiografoy.pdf

18. Consultado el 13 de junio de 2012. http://ww1. microchip.com/downloads/en/devicedoc/39632c.pdf

19. CCS., Inc. C Compiler Reference Manual. Brookfield, WI, USA: Custom Computer Services Inc; 2005. p. 152-153.

20. SENA., Technologis. Parani ESD-100/110/200/210 User guide. Version 1. 0. 2. Seoul, Korea: SENA Technologis Inc; 2006. p. 6-56.

21. Consultado el 13 de junio de 2012. http://www.java.com/ es/download/faq/whatis_j2me.xml

22. Consultado el 13 de junio de 2012. http://mobile. processing.org

23. Becerra-Luna B, Martínez-Memije R, Infante O. Monitor de frecuencia cardiaca mediante un teléfono móvil con el protocolo de comunicación Bluetooth. En: XXXIII Congreso Nacional de Ingeniería Biomédica 2010.

24. Polo PS, Orta VR, Flores ChP. Simulador portátil de electrocardiogramas. Rev Méx Ing Bioméd 1993;14:203-11. 